\title{
A predictive model of radiation-related fibrosis based on the radiomic features of magnetic resonance imaging and computed tomography
}

\author{
Jian Wang ${ }^{1}$, Rongjie Liu $^{2}$, Yu Zhao ${ }^{3}$, Chonnipa Nantavithya ${ }^{4}$, Hesham Elhalawani ${ }^{5}$, Hongtu Zhu ${ }^{6}$, \\ Abdallah Sherif Radwan Mohamed ${ }^{5}$, Clifton David Fuller ${ }^{5}$, Danita Kannarunimit ${ }^{4}$, Pei Yang ${ }^{7}$, Hong Zhu ${ }^{1}$ \\ ${ }^{1}$ Department of Oncology, Xiangya Hospital, Central South University, Changsha, China; ${ }^{2}$ Department of Statistics, Florida State University, \\ Tallahassee, Florida, USA; ${ }^{3}$ Unity Hospital, Rochester Region Health, Rochester, New York, USA; ${ }^{4}$ Department of Medicine, Chulalongkorn \\ University/King Chulalongkorn Memorial Hospital, Bangkok, Thailand; ${ }^{5}$ Department of Radiation Oncology, MD Anderson Cancer Center, \\ Houston, Texas, USA; ${ }^{6}$ Department of Biostatistics, University of North Carolina at Chapel Hill, Chapel Hill, NC, USA; ${ }^{7}$ Department of \\ Radiotherapy, Hunan Cancer Hospital, Affiliate Tumor Hospital of Xiangya Medical School, Central South University, Key Laboratory of \\ Translational Radiation Oncology of Hunan Province, Changsha, China \\ Contributions: (I) Conception and design: J Wang, P Yang, H Zhu; (II) Administrative support: H Zhu; (III) Provision of study materials or patients: \\ P Yang; (IV) Collection and assembly of data: J Wang; (V) Data analysis and interpretation: R Liu; (VI) Manuscript writing: All authors; (VII) Final \\ approval of manuscript: All authors. \\ Correspondence to: Hong Zhu. Department of Oncology, Xiangya Hospital, Central South University, Changsha 410008, China. Email: \\ zhuhong0719@126.com; Pei Yang. Department of Radiotherapy, Hunan Cancer Hospital, Affiliate Hospital of Xiangya Medical School, Central \\ South University, Key Laboratory of Translational Radiation Oncology of Hunan Province, Changsha 410013, China. Email: yangpei@hnca.org.cn.
}

Background: To establish a predictive model for the fibrotic level of neck muscles after radiotherapy by using radiomic features extracted from the magnetic resonance imaging (MRI) before and after radiotherapy and planning computed tomography (CT) in nasopharyngeal carcinoma patients.

Methods: A total of one hundred and eighty-six patients were finally enrolled in this study. According to the specific standard, all patients were divided into three different fibrosis groups. Regions of interests (ROI), including sternocleidomastoids (SCMs), trapezius (T), levator scapulae (LS), and scalenus muscles (S), were delineated manually and used for features extraction on IBEX. XGBoost, a machine learning algorithm, was used for the establishment of the prediction model. First, the patients were divided into training cohort (80\%) and testing cohort (20\%) randomly. Then the image features of CT or delta changes calculated from preand post-radiotherapy MRI images on each cohort constituted training and testing datasets. Then, based on the training dataset, a well-trained prediction model was produced. We used five-fold cross-validation to validate the predictive models. Afterward, the model performance was assessed on the 'testing' set and reported in terms of area under the receiver operating characteristic curve (AUC) under five scenarios: (I) only T1 sequence, (II) only T2 sequence, (III) only T1 post-contrast (T1 + C) sequence, (IV) Combination of all MRI sequences, (V) only CT.

Results: Most of the patients enrolled are male $(73.1 \%)$, mean age was 47 years, receiving concurrent chemo-radiotherapy as the primary treatment (90.9\%). By the end of the final follow-up, most of the patients were rated as mild fibrosis $(60.8 \%)$. We found the prediction model based on the CT image features outperform all MRI features with an AUC of 0.69 and accuracy of 0.65 . Contrarily, the model based on features from all MRI sequence showed lower AUC less than 0.5 and lower accuracy less than 0.6.

Conclusions: The prediction model based on CT radiomics features has better performance in the prediction of the grade of post-radiotherapy neck fibrosis. This might help guide radiotherapy treatment planning to achieve a better quality of life.

Keywords: Fibrosis; machine learning; nasopharyngeal carcinoma; quality of life 
Submitted Jan 28, 2020. Accepted for publication Jun 30, 2020.

doi: $10.21037 /$ tcr-20-751

View this article at: http://dx.doi.org/10.21037/tcr-20-751

\section{Introduction}

Nasopharyngeal carcinoma is relatively rare worldwide. In 2018, 129,079 new cases of this malignancy were diagnosed, accounting for $0.7 \%$ of all cancers (1). Still, nasopharyngeal carcinomas are commonly diagnosed in Asia, especially in China [the crude incidence rate was 3.26/100,000 (2)]. Radiotherapy remains the standard treatment for these generally radiosensitive tumors and current estimates of 5-year overall survival after radiotherapy range from $66 \%$ to $83 \%$ (3). Given these promising survival rates, oncologists increasingly have focused on the quality of life of their patients. Radiation-related fibrosis is a typical lateonset complication of radiotherapy (4). This sequela may not arise until a few years after the end of the treatment and may progress or deteriorate further over time $(4,5)$. Fibrosis can impair the functions of muscles in the head and neck and may thus restrict the opening of the mouth and jaw, motion in the shoulders, and rotation of the neck. Also, neck fibrosis may also cause cranial nerve palsy by compressing the hypoglossal nerve $(6,7)$. All of these restrictions can interfere with eating, speaking, driving, selfcare, and employment (5), severely impairing the quality of life. Radiation fibrosis is a multi-stage development process regulated by a variety of molecules, so it is difficult to design drugs that work at all stages. The current treatment strategies are mainly focused on limiting the aggravation of fibrosis, including topical emulsions (8), antioxidant therapies (9), hyperbaric oxygen therapy (10), adipose-derived stem cells (11) and some other therapies directly inhibiting the inflammatory mediators (12). The acupuncture and moxibustion therapy of traditional Chinese medicine also show a certain curative effect (13). However, the treatment options for radiation-related fibrosis are limited, and their therapeutic effects cannot sufficiently reverse the evolution and progression of fibrosis. Therefore, it is crucial to identify patients potentially at a high risk of fibrosis, as this will allow the application of preventive interventions to ensure optimal function and quality of life or minimize the side effects of treatment.

Fibrosis is characterized by an increase in tissue stiffness (i.e., loss of compliance), which can be detected by palpation. Accordingly, most studies have used hand palpation and clinician-based rating scales of fibrosis, including the Medical Research Council (MRC) (14), European Organization for Research and Treatment of Cancer/Radiation Therapy Oncology Group (EORTC/ RTOG) (15), and Late Effects in Normal Tissues/ Subjective, Objective, Management, and Analytic (LENT/ SOMA) scoring systems (16). However, these scales are inevitably subjective, semiquantitative (17), and prone to interobserver error. Other studies have applied quantitative mechanical methods (18), quantitative electrical methods $(19,20)$, ultrasound shear wave elastography $(21)$, and magnetic resonance imaging (MRI) $(22,23)$ to various parts of the body for fibrosis assessment. In the neck, tissue fibrosis may affect multiple tissue layers with considerable overlap. Currently, all of these changes cannot be detected using a single measurement technique, and even invasive biopsy is limited due to the ability to sample only specific microscopic points.

The field of radiomics is not based on information from images or single pathological tissue layers. Accordingly, this field differs from the traditional practice of subjecting medical images solely to visual interpretation (24). Radiomics exhibits tremendous promise as a comprehensive method of three-dimensional examination that enables the noninvasive profiling of multiple tissue layers (25). Nowadays, more and more researches begin to focus on the functional evaluation by using radiomics. A previous exploratory study succeeding in finding the relationship between the radiation dose to the masseter and the medial pterygoid and the variance of the MRI intensity of the radiation-induced trismus described by the radiomic textures (26). At the same time, a number of studies have shown the relationship between the image features and radiation-induced xerostomia and the integration of image features into the predictive model may improve the risk stratification of xerostomia (27-29). MRI is used widely in clinical workups for the pretreatment diagnosis and staging of nasopharyngeal carcinoma and as a conventional routine follow-up method. And every patient would perform planning CT scans before radiotherapy. In this study, therefore, we aimed to grope for if the radiomic features extracted from many types of MRI scans or CT could build a predictive model of radiation-related fibrosis. 


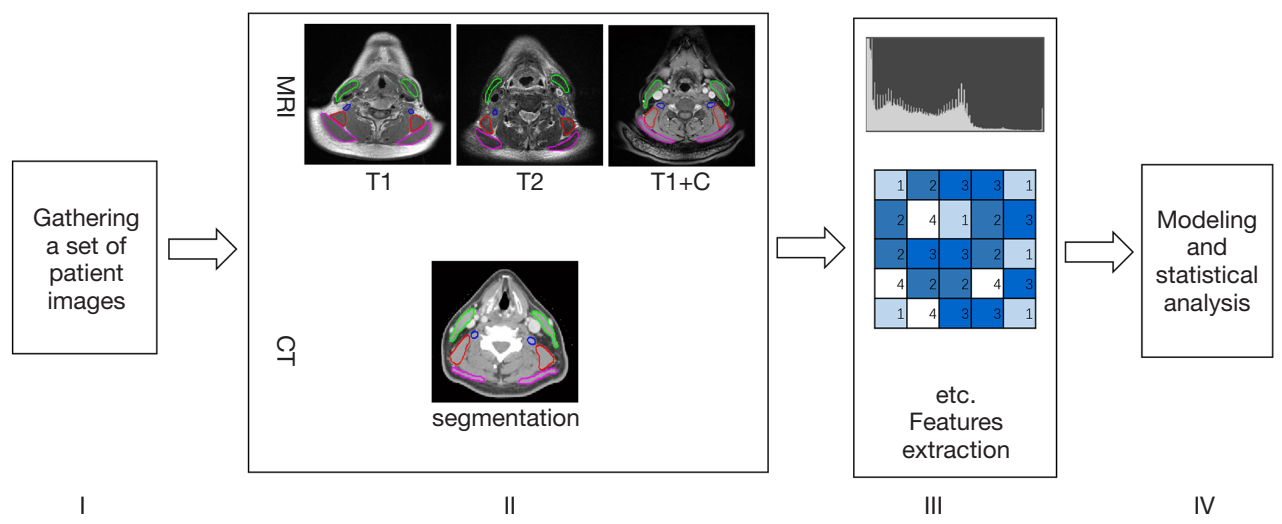

Figure $1 \mathrm{~A}$ workflow to produce a predictive model. These steps include gathering a set of patient images, segmentation of the region of interest (ROI) including four muscle, extracting a set of radiomics features from these ROIs, generating a predictive model and then perform the statistical analysis.

\section{Methods}

The workflow of this study is depicted in Figure 1. Specific contents and details in the process are described below.

\section{Patient cohort}

The study was conducted in accordance with the Declaration of Helsinki (as revised in 2013). The study was approved by Ethic Committee of the Hunan Cancer Hospital (No. 07 of 2020 Scientific Research Quick Review). The study is a retrospective study that examines the fait accompli of the past. Before the retrospective study, all the private information of patients will be anonymized, no direct contact with patients, no privacy of patients, the results of the study are only used for medical research, there is no risk to the patients included in the study. Based on the above, we applied for exemption from the informed consent of the subject and approved by the Ethic Committee.

For this study, we initially analyzed all MRI and CT data of 749 patients from the same institution in 2015, who received chemoradiation in 6-7 weeks. All MR images were obtained from a 1.5-T MRI system (GE sigma CV/ i) during routine clinical practice. All photos were axial scans with the field of view of $30 \mathrm{~cm}$, slice thickness of $5 \mathrm{~mm}$, and slice spacing of $1 \mathrm{~mm}$. CT images were acquired on GE LightSpeed RT (GEHW) with a peak tube voltage of $120 \mathrm{kVp}$ and exposure of $200 \mathrm{mAs}$. Images had $512 \times 512$ pixels, FOV of $550.0 \mathrm{~mm} \times 550.0 \mathrm{~mm}$, and a slice thickness of $5 \mathrm{~mm}$.

The final analysis included the images, clinical factors, and outcome data of the patients who met the following inclusion criteria: (I) initial treatment for pathologically confirmed nasopharyngeal carcinoma at a single institution in 2015; (II) no history of radiotherapy of neck and head because of other disease; (III) treated with static intensitymodulated radiation therapy (s-IMRT), step and shoot; (IV) availability of MR images collected at two points (before radiotherapy and up to 6 months post-treatment), including T1-weighted (T1), T1 post-contrast $(\mathrm{T} 1+\mathrm{C})$, and T2-weighted (T2) scans; (V) availability of planning CT images. Patients were excluded when they met the following criteria: (I) patients who could not be contacted at the follow-up time; (II) unavailability of MR images at each point or planning CT images; (III) poor image quality which is not sufficient for diagnosis and analysis. The specific screening process is shown in Figure 2.

\section{Clinical factors}

The patient's clinical parameters were obtained through a retrospective review and are presented systematically in Table 1. The median follow-up duration was 18 months (range, 11-22 months). The patients were asked about the presence of four symptoms: (I) discomfort in the neck (selfrated on a scale of $0-10$ ), (II) experience of late facial edema after treatment (yes or no, if yes, how long did it regress), (III) experience of upper limb pain after treatment (yes or no, if yes, the severity and is there any treatment), and (IV) restricted head rotation during activities of daily living (yes or no, if yes, the degree of limitation and how long does it last). Based on these symptoms and physical signs, all 


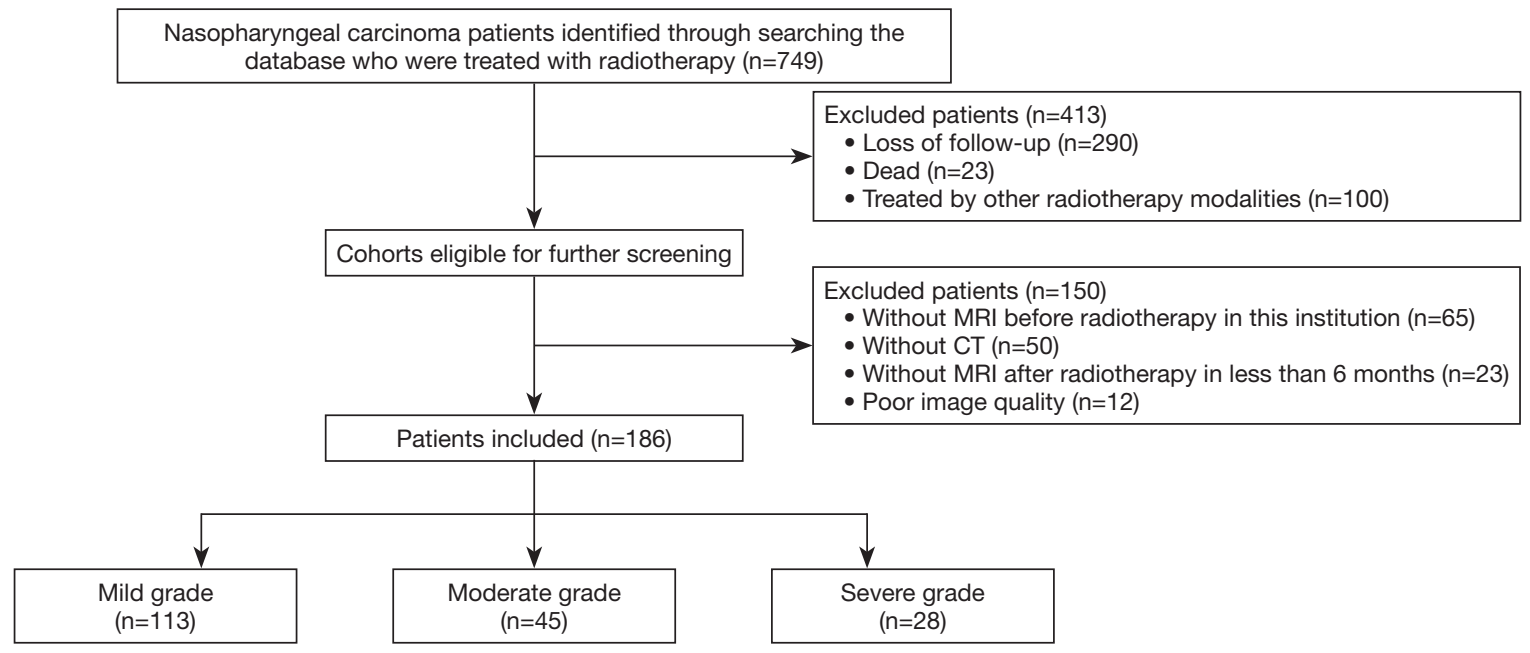

Figure 2 Flow diagram for patient selection in our study.

patients were divided into three groups: mild, moderate, and severe fibrosis. Specific standards are listed in Table 2.

\section{Lesion segmentation}

Lesion segmentation was performed using The Imaging Biomarker Explorer (IBEX) software package, version 1.0 (30). An experienced clinical oncologist used the IBEX software to contour the ROIs manually on each type of MRI sequence (T1, T1 + C, and T2) and CT for each patient. Later, the contoured ROI would be reviewed by another experienced radiologist. It has been reported that muscle fibrosis develops before skin fibrosis (31). Based on this theory, the ROI was drawn to include sternocleidomastoids (SCMs), trapezius (T), levator scapulae (LS), and scalenus muscles (S). The ROI was divided horizontally into two parts based on the level of the cricoid cartilage, which received a different radiation dose during radiotherapy. It is worth reminding that we contoured four ROIs above the cricoid cartilage on CT because of the loss of part images.

\section{Extraction and calculation of radiomic features}

IBEX was used to extract image features from pre- and post-radiotherapy MRI images or planning CT images. Categorization according to specific standards (Table 2) was used to label the image features like three different groups. As shown in Table 3, the textural features calculated in this study can be organized into 10 categories. Two processing methods were applied, including Resample Voxel Size and Butterworth Smooth (32), which lessened the image noise. Assigning different parameters to every textural feature, and a total of 190 texture features were acquired for each sequence of the MRI at every time points and 1,767 for CT. The magnitude of change of each feature was computed as follows: delta change $=($ post - pre $) /$ pre, being used for MRI feature modeling, where post and pre correspond to the measurements after and before radiation therapy, respectively. Changes from the first time to the second time in radiomics features, called delta-radiomics, have been proved to improved model for predicting the prognosis of patients combined with clinical factors and radiomic characteristics (33). On the meanwhile, features acquired on CT were directly used for modeling on the next step.

\section{Feature modeling}

In reality, many features have high noise and may lead to overfitting or classification errors in feature modeling. Not all image features can be conductive to grade the severity of neck fibrosis after radiotherapy. Features were selected for the prediction performance in terms of the AUC $(>0.6)$. Meanwhile, XGBoost, a Gradient Tree Boosting regularization form (34), could identify a subset of essential features to avoid feature redundancy for feature modeling in the course of calculation. Previous studies have reported that XGBoost showed lower test error rate and the larger AUC in comparison with logistic regression analysis and 
Table 1 Patient's clinical and dosimetric parameters

\begin{tabular}{|c|c|c|c|c|c|c|c|c|c|}
\hline Clinical factors & $\mathrm{N}=186$ & \multicolumn{4}{|c|}{ Training cohort } & \multicolumn{4}{|c|}{ Testing cohort } \\
\hline SEX & & & & & 0.972 & & & & 0.645 \\
\hline Male & 136 & 67 & 24 & 18 & & 17 & 6 & 4 & \\
\hline Female & 50 & 23 & 12 & 4 & & 6 & 3 & 2 & \\
\hline$\geq 65$ & 8 & 1 & 2 & 3 & & 1 & 1 & 0 & \\
\hline$<65$ & 178 & 89 & 34 & 19 & & 22 & 8 & 6 & \\
\hline T stage & & & & & 0.935 & & & & 0.852 \\
\hline $\mathrm{T} 1$ & 19 & 10 & 3 & 2 & & 2 & 1 & 1 & \\
\hline $\mathrm{T} 4$ & 48 & 22 & 9 & 7 & & 7 & 2 & 1 & \\
\hline N stage & & & & & 0.398 & & & & 0.714 \\
\hline NO & 4 & 1 & 1 & 1 & & 1 & 0 & 0 & \\
\hline $\mathrm{N} 1$ & 28 & 13 & 6 & 3 & & 3 & 1 & 2 & \\
\hline N2 & 112 & 58 & 20 & 11 & & 14 & 5 & 4 & \\
\hline N3 & 42 & 18 & 9 & 7 & & 5 & 3 & 0 & \\
\hline M stage & & & & & 0.03 & & & & 0.524 \\
\hline Mo & 178 & 88 & 36 & 18 & & 22 & 9 & 5 & \\
\hline IVa & 74 & 37 & 15 & 7 & & 9 & 4 & 2 & \\
\hline $\mathrm{IVb}$ & 8 & 2 & 0 & 4 & & 1 & 0 & 1 & \\
\hline Concurrent chemoradiotherapy & & & & & 0.641 & & & & 0.687 \\
\hline No & 17 & 9 & 4 & 1 & & 2 & 1 & 0 & \\
\hline Yes & 169 & 81 & 32 & 21 & & 21 & 8 & 6 & \\
\hline
\end{tabular}

The 7th American Joint Committee on Cancer (AJCC) TNM staging manual was used to stage the patients.

other machine learning approaches, including decision tree, random forest, and support vector machine (35). It leads us to propose using this method for the establishment of the prediction model of radiation-related fibrosis. First, the subjects included were divided into five folds which was consistent with the proportion of the three fibrotic groups in the overall cohort randomly. Then four folds constituted the training cohort, and the remaining one constituted the testing cohort. The modeling was done based on the selected image features delta changes on the MRI images 
Table 2 Categorization of 186 patients according to muscle fibrosis in the neck after treatment

\begin{tabular}{ll}
\hline Level & Conditions (meet any of the following) \\
\hline Mild & (I) Rating: 0-4 points \\
(II) No facial edema \\
(III) No upper limb pains \\
(IV) Neck activity is unrestricted \\
(I) Rating: 4-6 points \\
(II) Late facial edema (regress within 3 months) \\
(III) Mild upper limb pain (no treatment) \\
(IV) Neck activity is slightly limited \\
(I) Rating: 7-10 points \\
(II) Late facial edema, lasting more than 3 months \\
(III) Severe upper limb pain, or needing medical intervention \\
(IV) Neck activity is significantly limited or duration >6 months
\end{tabular}

Categorization into a specific group required the patient to meet any of the above criteria.

that were calculated in the previous step or the features extracted from CT on the cohort. Next, XGBoost was used to generate a well-trained prediction model based on the training image dataset. Though a lack of independent external validation, we do perform five-fold cross-validation (internal validation), which is identified to be an effective way to build patient-specific predictions without bias (36). Finally, we compared the prediction performance under five scenarios: (I) only the T1 sequence; (II) only the T2 sequence; (III) only the T1 + C sequence; (IV) combination all the MRI sequences; (V) only the CT. The performance of the predictive model was then assessed using the testing image set, and the results are reported in terms of the mean AUC.

\section{Results}

\section{Patients cohort and selected features}

As shown in Figure 2, lastly, a total of 186 patients were finally enrolled for further analysis. The patients include was divided into training cohort $(80.0 \%)$ and testing cohort (20\%) randomly. The patient sample was predominantly male $(73.1 \%)$, with a mean age of 47 years. The majority had locally advanced disease $(93.0 \%)$ and had received concurrent chemoradiotherapy as the primary treatment (90.9\%). By the end of the final follow-up, most patients were classified as having mild fibrosis $(60.8 \%)$. There are no significant differences among the fibrosis groups for all the factors except age $(\mathrm{P}=0.005)$ and metastasis stage $(\mathrm{P}=0.03)$ of the patients in the training cohort. Detailed clinical parameters are depicted in Table 1. As shown in Figure 3, fibrosis degree has nothing to do with the lymph node stage. We performed spearman relativity analysis between these two variables $\left(\mathrm{P}=0.613, \mathrm{r}_{\mathrm{s}}=0.029\right)$.

A total of 139 textures from T1 sequence, 138 features from $\mathrm{T} 2$ sequence and 157 features from $\mathrm{T} 1+\mathrm{C}$ sequence were used for feature modeling and 749 features for CT. As shown in Table 3, extracted features include firstorder, second-order and higher-order characteristic, which can be further grouped into ten categories. Shape features, Neighbor Intensity Difference features, Intensity Histogram Gaussian Fit features, Intensity histogram features, Intensity Direct features (37), Gray Level Cooccurrence Matrix features, Gray Level Run Length Matrix features (38), and Gradient Orient Histogram features (39) were enrolled in this study.

\section{Feature modeling}

The radiomics signatures based on MRI images or CT images performed well in predicting the degree of fibrosis after radiotherapy. Values of AUC, accuracy, sensitivity and specificity of the five scenarios are as shown in Table 4. When predicting three different fibrosis groups, we found the model based on the CT image features showed better 
Table 3 The features used in this study and related preprocessing methods

\begin{tabular}{|c|c|c|}
\hline Category & Feature & Preprocess \\
\hline \multirow{11}{*}{$\begin{array}{l}\text { Gradient } \\
\text { Orient } \\
\text { Histogram }\end{array}$} & Inter Quartile Range & Resample Voxel Size \\
\hline & & \\
\hline & & \\
\hline & Kurtosis & \\
\hline & Mean Absolute Deviation & \\
\hline & Median Absolute Deviation & \\
\hline & Percentile & \\
\hline & Percentile Area & \\
\hline & Quantile & \\
\hline & Range & \\
\hline & Skewness & \\
\hline \multirow{24}{*}{$\begin{array}{l}\text { Gray Level } \\
\text { Co-occurrence } \\
\text { Matrix } 25\end{array}$} & Auto Correlation & Resample Voxel Size \\
\hline & & \\
\hline & & \\
\hline & Cluster Prominence & \\
\hline & Cluster Shade & \\
\hline & Cluster Tendency & \\
\hline & Contrast & \\
\hline & Correlation & \\
\hline & Difference Entropy & \\
\hline & Dissimilarity & \\
\hline & Energy & \\
\hline & Entropy & \\
\hline & Homogeneity & \\
\hline & Homogeneity2 & \\
\hline & InformationMeasureCorr1 & \\
\hline & InformationMeasureCorr2 & \\
\hline & Inverse Diff Moment Norm & \\
\hline & Inverse Diff Norm & \\
\hline & Inverse Variance & \\
\hline & Max Probability & \\
\hline & Sum Average & \\
\hline & Sum Entropy & \\
\hline & Sum Variance & \\
\hline & Variance & \\
\hline
\end{tabular}

Table 3 (Continued)
Table 3 (Continued)

\begin{tabular}{lll}
\hline Category & Feature & Preprocess \\
\hline $\begin{array}{l}\text { Gray Level } \\
\text { Co-occurrence }\end{array}$ & Resample Voxel Size \\
Matrix 3 & &
\end{tabular}

Cluster Prominence

Cluster Shade

Cluster Tendency

Contrast

Correlation

Difference Entropy

Dissimilarity

Energy

Entropy

Homogeneity

Homogeneity2

InformationMeasureCorr1

InformationMeasureCorr2

Inverse Diff Moment Norm

Inverse Diff Norm

Inverse Variance

Max Probability

Sum Average

Sum Entropy

Sum Variance

Variance

Gray Level

Run Length

Gray Level

Nonuniformity

Resample Voxel Size,

Matrix25

High Gray Level Run

Emphasis

Long Run Emphasis

Long Run High Gray Level

Emphasis

Long Run Low Gray Level

Emphasis

Low Gray Level Run

Emphasis

Run Length Nonuniformity

Table 3 (Continued) 
Table 3 (Continued)

\begin{tabular}{|c|c|c|}
\hline Category & Feature & Preprocess \\
\hline \multirow{35}{*}{$\begin{array}{l}\text { Intensity } \\
\text { Direct }\end{array}$} & Run Percentage & \\
\hline & Short Run Emphasis & \\
\hline & Short Run High & \\
\hline & Gray Level Emphasis & \\
\hline & Short Run Low & \\
\hline & Gray Level Emphasis & \\
\hline & Energy & Resample Voxel Size \\
\hline & Energy Norm & \\
\hline & Global Entropy & \\
\hline & Global Max & \\
\hline & Global Mean & \\
\hline & Global Median & \\
\hline & Global Min & \\
\hline & Global Std & \\
\hline & Global Uniformity & \\
\hline & Inter Quartile Range & \\
\hline & Kurtosis & \\
\hline & Local Entropy Max & \\
\hline & Local Entropy Mean & \\
\hline & Local Entropy Median & \\
\hline & Local Entropy Min & \\
\hline & Local Entropy Std & \\
\hline & Local Range Max & \\
\hline & Local Range Mean & \\
\hline & Local Range Median & \\
\hline & Local Range Min & \\
\hline & Local Range Std & \\
\hline & Local Std Max & \\
\hline & Local Std Mean & \\
\hline & Local Std Median & \\
\hline & Local Std Min & \\
\hline & Local Std Std & \\
\hline & Mean Absolute Deviation & \\
\hline & Median Absolute Deviation & \\
\hline & Percentile & \\
\hline
\end{tabular}

Table 3 (Continued)

\begin{tabular}{|c|c|c|}
\hline Category & Feature & Preprocess \\
\hline & Quantile & \\
\hline & Range & \\
\hline & Root Mean Square & \\
\hline & Skewness & \\
\hline \multirow[t]{9}{*}{$\begin{array}{l}\text { Intensity } \\
\text { Histogram }\end{array}$} & Inter Quartile Range & $\begin{array}{l}\text { Resample Voxel Size, } \\
\text { Butterworth Smooth }\end{array}$ \\
\hline & Kurtosis & \\
\hline & Mean Absolute Deviation & \\
\hline & Median Absolute Deviation & \\
\hline & Percentile & \\
\hline & Percentile Area & \\
\hline & Quantile & \\
\hline & Range & \\
\hline & Skewness & \\
\hline \multirow{5}{*}{$\begin{array}{l}\text { Neighbor } \\
\text { Intensity } \\
\text { Difference } 25\end{array}$} & Busyness & $\begin{array}{l}\text { Resample Voxel Size, } \\
\text { Butterworth Smooth }\end{array}$ \\
\hline & Coarseness & \\
\hline & Complexity & \\
\hline & Contrast & \\
\hline & Texture Strength & \\
\hline \multirow{5}{*}{$\begin{array}{l}\text { Neighbor } \\
\text { Intensity } \\
\text { Difference } 3\end{array}$} & Busyness & $\begin{array}{l}\text { Resample Voxel Size, } \\
\text { Butterworth Smooth }\end{array}$ \\
\hline & Coarseness & \\
\hline & Complexity & \\
\hline & Contrast & \\
\hline & Texture Strength & \\
\hline \multirow[t]{8}{*}{ Shape } & Compactness 1 & - \\
\hline & Compactness2 & \\
\hline & Convex & \\
\hline & Convex Hull Volume & \\
\hline & Convex Hull Volume 3D & \\
\hline & Mass & \\
\hline & Max3D Diameter & \\
\hline & Mean Breadth & \\
\hline
\end{tabular}

Table 3 (Continued)

Table 3 (Continued) 
Table 3 (Continued)

\begin{tabular}{lll}
\hline Category & Feature & Preprocess \\
\hline & Number of Objects & \\
& Number of Voxel & \\
& Orientation & \\
& Roundness \\
& Spherical Disproportion \\
& Sphericity \\
& Surface Area \\
& Surface Area Density \\
& Volume \\
& Voxel Size \\
& Gaussian Amplitude & \\
Intensity & \\
Gistogram & Resample Voxel Size \\
& Gaussian Fit & \\
& Gaussian Mean \\
& Gaussian Std \\
&
\end{tabular}

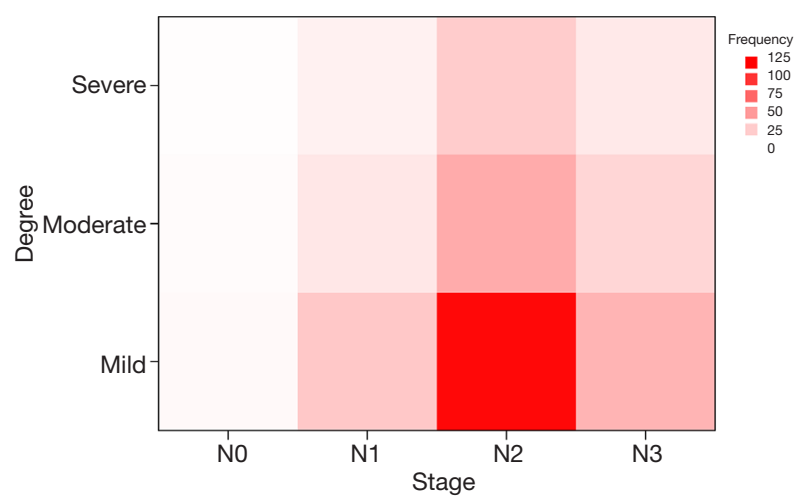

Figure 3 A hotspot map of the relationship between lymph node stage and the degree of fibrosis.

performance, with an AUC of 0.69 and accuracy of 0.65 compared to that models of $\mathrm{T} 1, \mathrm{~T} 2, \mathrm{~T} 1+\mathrm{C}$, and combine all three sequences show lower AUC (all is 0.49) and lower accuracy $(0.56,0.55,0.57,0.58)$.

\section{Discussion}

In this study, we developed predictive models of radiationrelated fibrosis based on the radiomic features of MRI and CT. These features exhibited predictive power to a certain extent. To our best knowledge, our group was the first to use the radiomic features of MRI and CT to predict the grade of radiation-related fibrosis on the neck. Additionally, we proposed a more practical standard for fibrosis level, including symptoms and physical signs.

Notably, the features extracted from CT outperformed all other feature changes from commonly used MRI scans, including $\mathrm{T} 1, \mathrm{~T} 2$, and $\mathrm{T} 1+\mathrm{C}$, in terms of AUC values and accuracy. Compared with CT, MRI has the advantage of showing soft tissue lesions better and providing musclespecific measurements. Researchers have previously identified a close relationship between MRI features and the severity of radiation-induced fibrosis (40). However, we did find that image features from CT have higher accuracy in predicting the degree of fibrosis after radiotherapy. Despite we only contoured four ROIs on CT images compared to eight ROIs on MRI, more features were extracted from CT. Besides, the type of acquisition noise, enhancement status, and image reconstruction algorithm have different effects on MRI imaging characteristics, especially in a retrospective study (41). To our knowledge, no standardized MRI method has been developed for the purpose of head and neck scanning or radiomics. Therefore, the effects of various MRI factors on the image data cannot yet be avoided. On the other hand, despite no existing studies have correlated clinically rated neck fibrosis with CT findings, previous studies have found textural image features extracted from CT are highly correlated with the severity of pulmonary fibrosis (42) and could discriminate between patients with and those without radiation pneumonitis (43). Moreover, the previous study has used CT texture changes for distinguishing radiation-induced fibrosis from tumor recurrence for lung cancer (44).

Despite the advantages of this approach, our research had some limitations. First, standard protocols for fibrosis grading included patient self-ratings, which inevitably led to subject bias. Second, the current radiomic analysis protocol involves complex computational steps with frequent human interactions and a potentially time-consuming analytical process and may be challenging to include in daily clinical practice. Currently, this post-processing process includes multiple steps and calculations and requires 
Table 4 The performance of the predictive model using the XGBoost

\begin{tabular}{|c|c|c|c|c|c|}
\hline Modality & AUC & Accuracy & Degree & Sensitivity (\%) & Specificity (\%) \\
\hline \multirow{2}{*}{ T1 } & 0.49 & 0.56 & (II) Moderate & 13.33 & 83.87 \\
\hline & - & - & (III) Severe & 3.33 & 96.20 \\
\hline \multirow[t]{2}{*}{ T2 } & - & - & (I) Mild & 84.04 & 6.71 \\
\hline & - & - & (III) Severe & 3.00 & 96.60 \\
\hline \multirow[t]{3}{*}{$\mathrm{T} 1+\mathrm{C}$} & - & - & (I) Mild & 81.35 & 19.29 \\
\hline & 0.49 & 0.57 & (II) Moderate & 17.33 & 83.34 \\
\hline & - & - & (III) Severe & 0.20 & 97.73 \\
\hline $\mathrm{T} 1+\mathrm{T} 2+\mathrm{T} 1+\mathrm{C}$ & - & - & (III) Severe & 3.00 & 96.58 \\
\hline \multirow[t]{3}{*}{ CT } & - & - & (I) Mild & 98.74 & 2.09 \\
\hline & 0.69 & 0.65 & (II) Moderate & 2.15 & 98.66 \\
\hline & - & - & (III) Severe & 0.00 & 100.00 \\
\hline
\end{tabular}

AUC, area under the curve; T1, T1-weighted scans; T1 + C, T1 post-contrast cans; T2, T2-weighted scans; CT, computed tomography.

approximately 60 minutes per patient (45). But we believe that as technological innovation and the optimization of the algorithm, it will be less time-consuming in the future. Moreover, our study did not distinguish between radiationinduced fibrosis and residual or recurrent tumor. The residual tumor remained after treatment in nearly $7-13 \%$ of nasopharyngeal carcinoma cases (46). A future prospective study should incorporate data from dynamic contrastenhanced MRI, the primary choice for the diagnosis of neck fibrosis after radiotherapy for nasopharyngeal carcinoma, given its ability to identify tumor residue, recurrence, and fibrosis (47). Finally, our analysis was based on a retrospective design and data from a single center. Our model requires validation through a prospective multicenter trial with a larger study cohort.

\section{Conclusions}

In conclusion, we constructed a predictive, non-invasive, inexpensive, and highly patient-specific model of radiationrelated fibrosis that does not affect existing clinical activities. This model requires further optimization, but it does contribute to the decision-making of the radiation treatment. Oncologists may use this model to compare the potential effects of different therapeutic regimens on the grade of radiation-related fibrosis and could thus individually tailor treatments to minimize the side reaction by adjusting the dose prescription on the neck. Furthermore, our model may inform studies of radiationrelated injuries in other body regions. In the future, we aim to develop the model further to enable direct predictions of the effects of radiation-related fibrosis on the quality of life.

\section{Acknowledgments}

Funding: This work was supported by Xiangya Hospital Clinical Research Project (grant number 2016L06), Beijing Xisike Clinical Oncology Research Foundation (grant number Y-HR2016-143), Scientific Research Program of Hunan Provincial Health Commission (B2019098) and Science and Technology Plan of Changsha Science and Technology Bureau (kq1801105).

\section{Footnote}

Data Sharing Statement: Available at http://dx.doi. org/10.21037/tcr-20-751 
Conflicts of Interest: All authors have completed the ICMJE uniform disclosure form (available at http://dx.doi. org/10.21037/tcr-20-751). The authors have no conflicts of interest to declare.

Ethical Statement: The authors are accountable for all aspects of the work in ensuring that questions related to the accuracy or integrity of any part of the work are appropriately investigated and resolved. The study was conducted in accordance with the Declaration of Helsinki (as revised in 2013). The study was approved by Ethic Committee of the Hunan Cancer Hospital (No. 07 of 2020 Scientific Research Quick Review). The study is a retrospective study that examines the fait accompli of the past, and all the private information of patients will be anonymized, no direct contact with patients, no privacy of patients. Based on the above, we applied for exemption from the informed consent of the subject and approved by the Ethic Committee.

Open Access Statement: This is an Open Access article distributed in accordance with the Creative Commons Attribution-NonCommercial-NoDerivs 4.0 International License (CC BY-NC-ND 4.0), which permits the noncommercial replication and distribution of the article with the strict proviso that no changes or edits are made and the original work is properly cited (including links to both the formal publication through the relevant DOI and the license). See: https://creativecommons.org/licenses/by-nc$\mathrm{nd} / 4.0 /$.

\section{References}

1. Bray F, Ferlay J, Soerjomataram I, et al. Global cancer statistics 2018: GLOBOCAN estimates of incidence and mortality worldwide for 36 cancers in 185 countries. CA Cancer J Clin 2018;68:394-424.

2. Chen WQ, Li H, Sun KX, et al. Report of Cancer Incidence and Mortality in China, 2014. Zhonghua Zhong Liu Za Zhi 2018;40:5-13.

3. Zhao Y, Shen L, Huang X, et al. Prognostic analysis of patients with locally advanced nasopharyngeal carcinoma following intensity modulated radiation therapy. Oncol Lett 2018;15:4445-50.

4. Hirota $\mathrm{S}$, Tsujino K, Oshitani T, et al. Subcutaneous fibrosis after whole neck irradiation. Int J Radiat Oncol Biol Phys 2002;52:937-43.

5. Zheng Y, Han F, Xiao W, et al. Analysis of late toxicity in nasopharyngeal carcinoma patients treated with intensity modulated radiation therapy. Radiat Oncol 2015;10:17.

6. Kong L, Lu JJ, Liss AL, et al. Radiation-Induced Cranial Nerve Palsy: a Cross-Sectional Study of Nasopharyngeal Cancer Patients After Definitive Radiotherapy. Int J Radiat Oncol Biol Phys 2011;79:1421-7.

7. Lin YS, Jen YM, Lin JC. Radiation related cranial nerve palsy in patients with nasopharyngeal carcinoma. Cancer 2002;95:404-9.

8. Menêses AG, Reis PEDD, Guerra ENS, et al. Use of trolamine to prevent and treat acute radiation dermatitis: a systematic review and meta-analysis. Rev Lat Am Enfermagem 2018;26:e2929.

9. Lin SL, Chen RH, Chen YM, et al. Pentoxifylline attenuates tubulointerstitial fibrosis by blocking Smad3/4activated transcription and profibrogenic effects of connective tissue growth factor. J Am Soc Nephrol 2005;16:2702-13.

10. Borab Z, Mirmanesh MD, Gantz M, et al. Systematic Review of Hyperbaric Oxygen Therapy for the Treatment of Radiation-Induced Skin Necrosis. J Plast Reconstr Aesthet Surg 2017;70:529.

11. Borovikova AA, Ziegler ME, Banyard DA, et al. AdiposeDerived Tissue in the Treatment of Dermal Fibrosis. Ann Plast Surg 2018;80:297-307.

12. Xavier S, Piek E, Fujii M, et al. Amelioration of Radiationinduced Fibrosis. J Biol Chem 2004;279:15167-76.

13. Yang Y, Guo H, Wang L. Clinical observation on acupuncture therapy and massage therapy of fibrosis in patients with nasopharyngeal carcinoma after radiotherapy. China Practical Medicine 2010;29:27-28.

14. Dische $S$. The uniform reporting of treatment-related morbidity. Semin Radiat Oncol 1994;4:112.

15. Cox JD, Stetz J, Pajak TF. Toxicity criteria of the Radiation Therapy Oncology Group (RTOG) and the European Organization for Research and Treatment of Cancer (EORTC). Int J Radiat Oncol Biol Phys 1995;31:1341-6.

16. Pavy JJ, Denekamp J, Letschert J, et al. EORTC Late Effects Working Group. Late effects toxicity scoring: the SOMA scale. Int J Radiat Oncol Biol Phys 1995;31:1043-7.

17. Leung SF, Zheng Y, Choi CY, et al. Quantitative measurement of post-irradiation neck fibrosis based on the young modulus: description of a new method and clinical results. Cancer 2002;95:656-62.

18. Guy RH. Handbook of non-invasive methods and the skin. J Control Release 1995;1:102.

19. Paulsen KD, Osterman KS, Hoopes PJ. In vivo 
electrical impedance spectroscopic monitoring of the progression of radiation-induced tissue injury. Radiat Res 1999;152:41-50.

20. Osterman KS, Hoopes PJ, DeLorenzo C, et al. Noninvasive assessment of radiation injury with electrical impedance spectroscopy. Phys Med Biol 2004;49:665-83.

21. Bercoff J, Tanter M, Fink M. Supersonic shear imaging: a new technique for soft tissue elasticity mapping. IEEE Trans Ultrason Ferroelectr Freq Control 2004;51:396-409.

22. Wang J, Malik N, Yin M, et al. Magnetic resonance elastography is accurate in detecting advanced fibrosis in autoimmune hepatitis. World J Gastroenterol 2017;23:859

23. Hu G, Zhang X, Liang W, et al. Assessment of liver fibrosis in rats by MRI with apparent diffusion coefficient and $\mathrm{T} 1$ relaxation time in the rotating frame. J Magn Reson Imaging 2016;43:1082-9.

24. Gillies RJ, Kinahan PE, Hricak H. Radiomics: Images are more than pictures, they are data. Radiology 2016;278:563-77.

25. Lambin P, Rios-Velazquez E, Leijenaar R, et al. Radiomics: Extracting more information from medical images using advanced feature analysis. Eur J Cancer 2012;48:441-6.

26. Thor M, Tyagi N, Hatzoglou V, et al. A magnetic resonance imaging-based approach to quantify radiation-induced normal tissue injuries applied to trismus in head and neck cancer. Phys Imaging Radiat Oncol 2017;1:34-40.

27. Gabryś HS, Buettner F, Sterzing F, et al. Design and Selection of Machine Learning Methods Using Radiomics and Dosiomics for Normal Tissue Complication Probability Modeling of Xerostomia. Front Oncol 2018;8:35.

28. van Dijk LV, Thor M, Steenbakkers RJHM, et al. Parotid gland fat related magnetic resonance image biomarkers improve prediction of late radiation-induced xerostomia. Radiother Oncol 2018;128:459-66.

29. Sheikh K, Lee SH, Cheng Z, et al. Predicting acute radiation induced xerostomia in head and neck Cancer using MR and CT Radiomics of parotid and submandibular glands. Radiat Oncol 2019;14:131.

30. Zhang L, Fried DV, Fave XJ, et al. IBEX: an open infrastructure software platform to facilitate collaborative work in radiomics. Med Phys 2015;42:1341-53.

31. Li J, Wang RS, Liu WQ, et al. Cervical fibrosis and its influencing factors in patients with nasopharyngeal carcinoma after radiotherapy. Chin J Radiol Med Protection 2005;3:253-5.
32. Dogra A, Bhalla P. Image Sharpening By Gaussian And Butterworth High Pass Filter. Biomed Pharmacol J 2014;2:707-13.

33. Fave X, Zhang L, Yang J, et al. Delta-radiomics features for the prediction of patient outcomes in non-small cell lung cancer. Sci Rep 2017;7:588.

34. Liu L, Yu Y, Fei Z, et al. An interpretable boosting model to predict side effects of analgesics for osteoarthritis. BMC Syst Biol 2018;12:105.

35. Lee HC, Yoon HK, Nam K, et al. Derivation and validation of machine learning approaches to predict acute kidney injury after cardiac surgery. J Clin Med 2018;7:322.

36. Simon RM, Subramanian J, Li MC, et al. Using crossvalidation to evaluate predictive accuracy of survival risk classifiers based on high-dimensional data. Brief Bioinform 2011;12:203-14.

37. Haralick RM, Shanmugam K, Dinstein I. Textural features for image classification. IEEE 1973;6:610-1.

38. Galloway MM. Texture analysis using gray level run lengths. Comput Graph Image Process 1975;2:172-9.

39. Li B, Cheng K, Yu Z. Histogram of Oriented Gradient Based Gist Feature for Building Recognition. Comput Intell Neurosci 2016;2016:6749325.

40. Hoeller U, Bonacker M, Bajrovic A, et al. Radiationinduced plexopathy and fibrosis: Is magnetic resonance imaging the adequate diagnostic tool? Strahlenther Onkol 2004;180:650-4.

41. Qian L, Zhaoxiang Y. The application of imaging in tumor research. Chin J Oncol 2018;11:801-4.

42. Caban JJ, Yao J, Bagci U, et al. Monitoring pulmonary fibrosis by fusing clinical, physiological, and computed tomography features. Conf Proc IEEE Eng Med Biol Soc 2011;2011:6216-9.

43. Cunliffe A, Armato SG, Castillo R, et al. Lung Texture in Serial Thoracic Computed Tomography Scans: Correlation of Radiomics-based Features With Radiation Therapy Dose and Radiation Pneumonitis Development. Int J Radiat Oncol Biol Phys 2015;91:1048-56.

44. Mattonen SA, Palma DA, Haasbeek CJA, et al. Early prediction of tumor recurrence based on CT texture changes after stereotactic ablative radiotherapy (SABR) for lung cancer. Med Phys 2014;41:033502.

45. Kickingereder P, Neuberger U, Bonekamp D, et al. Radiomic subtyping improves disease stratification beyond key molecular, clinical, and standard imaging characteristics in patients with glioblastoma. Neuro Oncology 2018;20:848-57.

46. Su D, Jin G, Xie D, et al. Identification of local recurrence 
and radiofibrosis by computed tomography perfusion on nasopharyngeal carcinoma after radiotherapy. Can Assoc Radiol J 2010;61:265-70.

47. Mao J, Shen J, Yang Q, et al. Intravoxel incoherent motion

Cite this article as: Wang J, Liu R, Zhao Y, Nantavithya C, Elhalawani H, Zhu H, Mohamed ASR, Fuller CD, Kannarunimit D, Yang P, Zhu H. A predictive model of radiation-related fibrosis based on the radiomic features of magnetic resonance imaging and computed tomography. Transl Cancer Res 2020;9(8):4726-4738. doi: 10.21037/tcr-20-751
MRI in differentiation between recurrent carcinoma and postchemoradiation fibrosis of the skull base in patients with nasopharyngeal carcinoma. J Magn Reson Imaging 2016;44:1556-64. 